\title{
More but not less uncertainty makes adult humans' tool selections more similar to those reported with crows
}

\author{
Francisco J. Silva $・$ Kathleen M. Silva
}

Published online: 28 February 2012

(C) Psychonomic Society, Inc. 2012

\begin{abstract}
In this study, we examined whether adult humans' tool selections in a stick-and-tube problem might resemble previously published results of crows' selections if people had more experience solving the problem or were presented with a more ambiguous problem. In Experiments $1 \mathrm{a}$ and $1 \mathrm{~b}$, when given multiple opportunities to select a stick from a set of 10 to retrieve a candy located either 8 or $16 \mathrm{~cm}$ from the opening of a tube, the participants always selected a stick that was long enough to retrieve the candy; however, they did not generally select either the stick whose length matched the object's distance or the longest stick in the set - two outcomes reported in studies with crows. In Experiment 2, participants who were allowed only a brief period of time to study the problem selected a longer stick than did participants allowed unlimited time to do the same. However, only when the candy's distance was $16 \mathrm{~cm}$ did these people reliably select the longest stick in the set. It seems that increasing, but not decreasing, people's uncertainty about a problem can make humans' tool selections more similar to those reported with crows.
\end{abstract}

Keywords Experience · Uncertainty · Tool use · Humans · Crows

When presented with a set of 10 stick-tools that varied in length, two captive New Caledonian crows (Corvus moneduloides) most often attempted to retrieve food in a horizontal pipe by selecting the longest stick in the set and, less

\section{F. J. Silva $(\bowtie) \cdot$ K. M. Silva}

Department of Psychology, University of Redlands,

P.O. Box 3080, 1200 East Colton Avenue,

Redlands, CA 92373-0999, USA

e-mail: francisco_silva@redlands.edu often, a stick whose length was exactly the same as (i.e., matched) the distance over which the food had to be retrieved (Chappell \& Kacelnik, 2002). That the crows rarely selected a tool that was too short to retrieve the food suggested that they had an understanding of physical causality that included the ability to identify the distance over which a desired object had to be retrieved, to identify a tool whose length matched or exceeded this distance, and to use this tool to retrieve the object.

The generality of Chappell and Kacelnik's (2002) findings were studied by Hunt, Rutledge, and Gray (2006), who found that New Caledonian crows tested in a naturalistic setting generally selected, as their first and usually unsuccessful response, twigs or leaf stems whose length did not match the depth of a hole (deep or shallow) from which they had to retrieve food. But in a study of this same species in their natural environment, Bluff, Troscianko, Weir, Kacelnik, and Rutz (2010) found that the crows selected tools whose lengths were positively correlated with, though did not reliably match, the depth of the holes from which they fished for larvae. Using a different species, Tebbich and Bshary (2004) found that woodpecker finches (Cactospiza pallida) selected sticks whose lengths generally exceeded the distance over which the food had to be retrieved. While these findings with avian species have conflicted as to the typical length (e.g., matching, longest, or longer than necessary) of the tool chosen on the first trial, a consistent finding has been that, with experience, birds successfully retrieve an object with a tool of appropriate length (Emery \& Clayton, 2009).

In contrast to avian species, humans rarely select a tool whose length matches the distance to a desired object or the longest tool in a set (Silva \& Silva, 2010). Using a task that was similar to the one used with crows (e.g., Chappell \& Kacelnik, 2002), adult humans who had one opportunity to 
select a stick from a set of 10 to retrieve a candy placed in a horizontal tube generally selected a stick that was a few centimeters longer than the distance over which the candy had to be retrieved, and then they held this stick some distance from its end (Silva \& Silva, 2010). This resulted in a stick with a shorter useable or working length. Importantly, the working length of the stick also did not match the candy's distance. We suggested that these results occurred because the participants, who did not have explicit experience with the tools and task, chose a stick that they guessed would be long enough to reach the object but not so long as to be unnecessarily unwieldy. In this sense, the participants' choices may have reflected some degree of uncertainty about the task and how to use the tools to retrieve the object. This account leads to the overarching question of the present study: To what extent are people's tool selections affected by their uncertainty about the tools and task?

We addressed this question in three experiments. In Experiments $1 \mathrm{a}$ and $1 \mathrm{~b}$, we attempted to decrease participants' uncertainty about the tools and task by allowing them to select tools and retrieve an object across several trials. In this circumstance, increased experience should reduce uncertainty about the tools and about how to use them to retrieve the object from the tube. In Experiment 2, we attempted to increase uncertainty about the tools and task. Although we may decrease uncertainty by allowing participants multiple trials to select tools and retrieve an object (e.g., Exps. 1a and 1b), we cannot increase uncertainty by giving participants less than one trial to do the same. However, we can approximate giving participants less than one trial by shortening the duration of a trial (Exp. 2).

Although an abundance of research has focused on tool use in nonhuman primates, avian species, and human children, adult humans' understanding of physical causality has been largely assumed (Silva, Page, \& Silva, 2005; see, e.g., Povinelli, 2000). Identifying the conditions that produce similar tool-use performance in humans and other animals might help identify similar underlying mechanisms. In the comparative study of physical causality, the use of adult humans and simple problems allows us to manipulate the features of a problem or a procedure while knowing that any changes to the participant's behavior are unlikely to reflect the absence of an understanding of the critical features of the problem, especially as they relate to abstract concepts such as "gravity" and "transfer of force" (cf. Povinelli, 2000). In this regard, studies of adult humans help provide a context in which to interpret the behavior of nonhumans presented with similar tasks (see, e.g., Silva et al., 2005; Silva \& Silva, 2006).

\section{Experiment 1a}

To retrieve out-of-reach objects, people choose tools that are longer than the distance over which an object must travel
(Silva \& Silva, 2010). When people have no experience with the tools and task and are allowed only a single trial to select a tool, choosing the longer-than-necessary stick is a safe alternative, in that it is likely to lead to success and requires relatively little cognitive effort in terms of foreseeing how the stick will be held and used, estimating the object's distance, and evaluating whether a particular tool is long enough to reach the object. To the extent that people's tool selections reflect some uncertainty about the tools (e.g., judging their lengths) and the task (e.g., the ease of retrieving the object), it should be possible to reduce this uncertainty by giving people experience with the tools and task. With repeated opportunities to select a tool and retrieve an object, might adult humans come to select a tool whose length more closely approximates the distance over which the object is to be retrieved, a result observed with some laboratory crows (e.g., Chappell \& Kacelnik, 2002), and sometimes with wild crows at natural foraging sites (e.g., Bluff et al., 2010)?

\section{Method}

Participants A group of 20 undergraduate students attending a liberal arts college in southern California volunteered for the study and received partial course credit for their involvement.

Materials Ten 0.25 -cm-diameter wooden dowels, ranging in length from 8 to $26 \mathrm{~cm}$, served as tools. The participants used one of the sticks to retrieve a candy (an M\&M) from a transparent tube that was $4 \mathrm{~cm}$ in diameter, $30 \mathrm{~cm}$ long, and open at one end. These materials comprised the same number of sticks, lengths of sticks, and tube design used by Chappell and Kacelnik (2002). The tube was secured to the surface of a table. The experimenter placed the candy either 8 or $16 \mathrm{~cm}$ from the open end of the tube, which sometimes faced the left side of the table and sometimes the right, as viewed from where the participant stood while she or he evaluated which stick to select. The sticks (dowels) were laid flat on the table, centered and perpendicular to the tube. Each stick was separated from any adjacent stick by $3 \mathrm{~cm}$, and the sticks were arranged so that the bottom ends were aligned. The position of any individual stick varied randomly between participants.

A sheet of $30 \times 30 \mathrm{~cm}, 1-\mathrm{cm}$-thick privacy glass was positioned $8 \mathrm{~cm}$ above the tube with the candy. Privacy glass is opaque until it is switched on, at which time it clears instantly and remains transparent until switched off, at which time it instantly becomes opaque again. The privacy glass was on (i.e., in its transparent state) throughout the experiment. A schematic of the setup is shown in Fig. 1. 


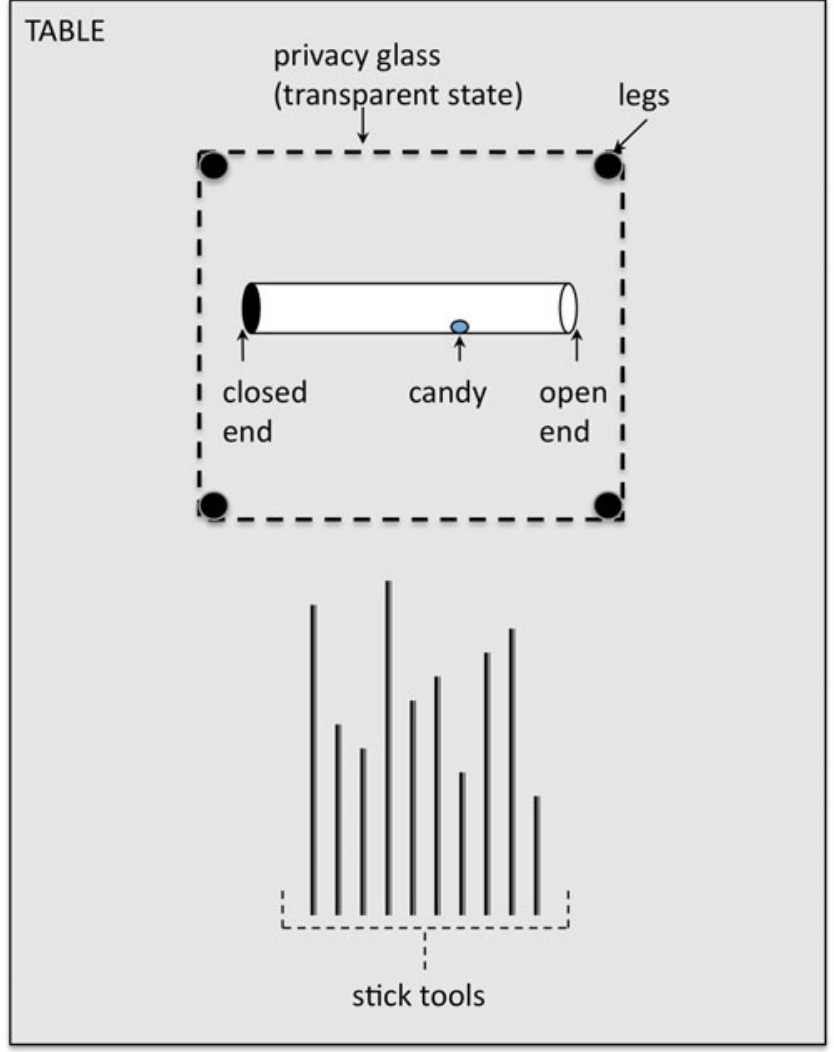

Participant Stood Here When Selecting Tool

Fig. 1 A schematic of the stick-and-tube task used in Experiments 1a, $1 \mathrm{~b}$, and 2 (not drawn to scale). The display of the tools was randomized across participants. The distance of the food from the open end of the tube was either 8 or $16 \mathrm{~cm}$. For Experiments 1a and 1b, the privacy glass was always in its transparent state. For Experiment 2, the state of the privacy glass (transparent vs. opaque) varied as described in this experiment's procedure

Procedure The participants were tested individually. The experimenter set up the tube and sticks before a participant entered the testing room. The experiment began with the experimenter asking a participant to enter the testing room and to stand in front of the tube and the sticks, while the experimenter read the instructions. The candy was not yet in the tube. The experimenter told the participants that they were participating in an experiment about how people use tools to solve problems, that they would use one of the sticks to retrieve a candy from the tube, and that they would be asked to do this several times. The experimenter then asked the participant to leave the room while the candy was placed in the tube. When a participant reentered the room, she or he was instructed to stand in the same place as when the instructor had explained the experiment and then to select a stick. Once a participant selected a stick, he or she could move around to any side of the table to retrieve the candy.

Each participant completed two phases, each of which consisted of four trials. In one phase, the candy was $8 \mathrm{~cm}$ from the open end of the tube; in the other, the candy was $16 \mathrm{~cm}$ from the open end. The order (8- vs. 16-cm distance) and the orientation of the opening (left vs. right) were counterbalanced across participants. Thus, for 10 participants (Group 8-16), the candy was placed $8 \mathrm{~cm}$ from the opening of the tube during the first four trials, and $16 \mathrm{~cm}$ from the opening during the next four trials. For half of the participants in this group, the opening of the tube faced right. For the 10 other participants (Group 16-8), the candy was placed $16 \mathrm{~cm}$ from the opening of the tube during the first four trials, and $8 \mathrm{~cm}$ from the opening during the last four trials. For half of the participants in this group, the opening of the tube faced right.

The experimenter recorded the stick chosen by each participant and whether the candy was successfully retrieved. The experimenter then asked the participant to leave the room while the candy and the sticks were prepared for the next trial. The orientation of the open end of the tube (left or right) did not change between trials. Also, because our goal was to examine the effects of experience on people's tool selections, the arrangement of the sticks on the table was held constant across trials for any individual participant. The specific arrangement of the sticks, however, varied randomly across participants. After the eighth trial, the participants answered two open-ended questions asking them to explain how their experience retrieving the candy affected the stick they chose when the object was near the open end of the tube (the $8-\mathrm{cm}$ distance) and when the object was farther from the end of the tube (i.e., the $16-\mathrm{cm}$ distance).

Unless otherwise stated, the results were analyzed with an analysis of variance (ANOVA) consisting of two between-groups factors (order of the candy's distance and orientation of the opening of the tube) and two within-groups factors (candy's distance and trials). Tukey's honestly significant difference (HSD) test was used for the post hoc analyses. For all analyses, $\alpha=.05$ was used to determine statistical significance.

\section{Results and discussion}

Every participant retrieved the candy on every trial. Table 1 shows the distribution of the participants' tool selections. Had the participants selected a stick at random, these selections would have been equally distributed across the 10 sticks. That did not occur. Instead, when the candy's distance was $8 \mathrm{~cm}$ from the open end of the tube, the participants generally selected sticks that were 14-20 cm long. When the candy's distance was $16 \mathrm{~cm}$, the participants most often selected sticks that were $22-26 \mathrm{~cm}$ long.

Figure 2 shows the average lengths of the sticks selected by the participants on each trial when the candy was 8 or 
Table 1 Frequency distribution of sticks selected in Experiment 1a

\begin{tabular}{|c|c|c|c|c|c|c|c|c|c|c|c|}
\hline \multirow[t]{2}{*}{ Candy Distance } & \multirow[t]{2}{*}{ Trial } & \multicolumn{10}{|c|}{ Stick Length (cm) } \\
\hline & & 8 & 10 & 12 & 14 & 16 & 18 & 20 & 22 & 24 & 26 \\
\hline \multicolumn{12}{|l|}{ Group 8-16 } \\
\hline \multirow[t]{4}{*}{$8 \mathrm{~cm}$} & 1 & 0 & 0 & 1 & 2 & 3 & 2 & 0 & 0 & 0 & 2 \\
\hline & 2 & 0 & 0 & 0 & 3 & 2 & 1 & 1 & 1 & 2 & 0 \\
\hline & 3 & 0 & 0 & 0 & 3 & 2 & 1 & 3 & 1 & 0 & 0 \\
\hline & 4 & 0 & 0 & 0 & 1 & 3 & 0 & 4 & 1 & 1 & 0 \\
\hline \multirow[t]{4}{*}{$16 \mathrm{~cm}$} & 5 & 0 & 0 & 0 & 0 & 1 & 1 & 1 & 2 & 1 & 4 \\
\hline & 6 & 0 & 0 & 0 & 0 & 0 & 0 & 1 & 2 & 1 & 6 \\
\hline & 7 & 0 & 0 & 0 & 0 & 0 & 0 & 1 & 2 & 3 & 4 \\
\hline & 8 & 0 & 0 & 0 & 0 & 0 & 0 & 1 & 4 & 1 & 4 \\
\hline \multicolumn{12}{|l|}{ Group 16-8 } \\
\hline \multirow[t]{4}{*}{$16 \mathrm{~cm}$} & 1 & 0 & 0 & 0 & 0 & 0 & 1 & 0 & 4 & 4 & 1 \\
\hline & 2 & 0 & 0 & 0 & 0 & 1 & 0 & 0 & 5 & 3 & 1 \\
\hline & 3 & 0 & 0 & 0 & 0 & 0 & 0 & 0 & 5 & 5 & 0 \\
\hline & 4 & 0 & 0 & 0 & 0 & 0 & 2 & 0 & 5 & 3 & 0 \\
\hline \multirow[t]{4}{*}{$8 \mathrm{~cm}$} & 5 & 0 & 0 & 2 & 1 & 4 & 1 & 1 & 1 & 0 & 0 \\
\hline & 6 & 0 & 0 & 0 & 2 & 5 & 1 & 1 & 1 & 0 & 0 \\
\hline & 7 & 0 & 1 & 0 & 5 & 0 & 1 & 3 & 0 & 0 & 0 \\
\hline & 8 & 0 & 1 & 0 & 5 & 0 & 1 & 2 & 1 & 0 & 0 \\
\hline
\end{tabular}

$16 \mathrm{~cm}$ from the open end of the tube. There was no overall difference between Groups $8-16$ and $16-8[F(1,16)=2.80$, $p=.113]$. When the candy's distance was $8 \mathrm{~cm}$, the length of the average stick selected by the participants in Group 8-16 was $18.0 \mathrm{~cm}(S D=3.71)$; for Group $16-8$, this length was $16.2 \mathrm{~cm}(S D=3.16)$. This difference in the lengths of the sticks was not statistically significant $(p=.227)$. When the candy's distance was $16 \mathrm{~cm}$, the length of the average stick selected by Group 8-16 was $23.7 \mathrm{~cm}(S D=2.64)$; for Group $16-8$, this

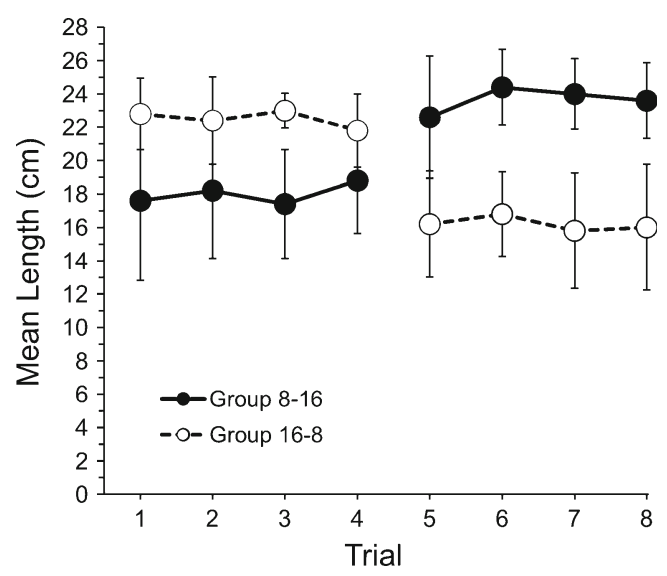

Fig. 2 Mean lengths of the sticks selected in Experiment 1a for each group across all trials. The error bars show standard deviations. The distance of the candy was changed before the fifth trial; for Group 8-16, the candy's distance was doubled from 8 to $16 \mathrm{~cm}$, and for Group 16-8, the candy's distance was halved from 16 to $8 \mathrm{~cm}$ length was $22.5 \mathrm{~cm}(S D=2.06)$. This difference was also not statistically significant $(p=.587)$. The orientation of the opening of the tube had no effect on participants' tool selections $[F(1,16)=0.50, p=.489]$, and there were no differences across trials $[F(3,48)=0.76, p=.523]$ or interactions among any combinations of the factors $[F \mathrm{~s}(1,16)<2.61, p \mathrm{~s}>.126$, and $\left.F_{\mathrm{s}}(3,48)<1.34, p \mathrm{~s}>.274\right]$. Thus, for both groups at both of the candy's distances, tool selections did not change across trials.

The only difference shown in Fig. 2 is that the average length of the sticks chosen by the participants was shorter when the candy's distance was $8 \mathrm{~cm}$ than when it was $16 \mathrm{~cm}$. When the distance was $8 \mathrm{~cm}$, the length of the average stick chosen was $17.1 \mathrm{~cm}(S D=3.54)$; when the distance was $16 \mathrm{~cm}$, the length of the average stick was $23.1 \mathrm{~cm}$ $(S D=2.42)$. This difference was statistically significant $[F(1,16)=88.56, p<.001]$.

Although there were no differences in the average lengths of the sticks selected across trials, perhaps there were differences across trials if we were to look only at whether participants selected a stick that was different from the one they had selected on the previous trial. This measure of "tool swapping" (Wimpenny, Weir, Clayton, Rutz, \& Kacelnik, 2009) provides information about whether participants were experimenting with different tools. To obtain this measure, we noted which stick participants chose on a particular trial (e.g., Trial 1) and whether they selected a different stick on the next trial (e.g., Trial 2). If a different stick was selected, this choice was recorded as a "1"; if a new stick was not selected, this 
choice was recorded as a " 0 ." We looked at whether the sticks on Trials 1 versus 2, 2 versus 3, 3 versus 4, 5 versus 6,6 versus 7 , and 7 versus 8 were the same or different. (We did not look at Trials 4 vs. 5 because between these trials the candy's distance was changed from 8 to $16 \mathrm{~cm}$, or vice versa.) This analysis produced a tally of how many participants changed sticks between the trials listed above, and it allowed us to answer the question of whether the participants' unfamiliarity with the task and the tools made it more likely that they would try different sticks during earlier versus later trials.

Collapsing across the candy's two distances, the results showed that on Trials 2, 3, and $4,80 \%, 50 \%$, and $55 \%$ of the participants, respectively, selected a different stick from the one they had chosen on the previous trial. The candy's distance from the opening of the tube was changed before the fifth trial. Then, on Trials 6,7 , and $8,50 \%, 60 \%$, and $40 \%$ of the participants selected a different stick from the one they had chosen on the previous trial. It seems that the participants were most likely to select a different stick between Trials 1 and 2, and then to swap sticks less often across trials. Cochran's $Q$ test confirmed that there was a statistically significant difference in the percentages of participants who selected a different stick across the six pairs of trials noted above $[Q(5)=11.40, p=.04]$.

The major findings of this experiment showed that the length of the stick selected by the participants (a) was influenced by the candy's distance from the opening of the tube, (b) was unaffected by having retrieved candies at another distance, and (c) did not change as a function of experience retrieving the candy. The first result replicates findings that people generally select a tool that is longer than the distance over which a desired object has to be retrieved (Silva \& Silva, 2010), a result that has also been observed in crows. More specifically, as the distance of a desired object (food) increased, the length of the tool selected by the crows to retrieve the food also increased (Chappell \& Kacelnik, 2002; Wimpenny et al., 2009). The second result showed no carryover effects of having retrieved candies $8 \mathrm{~cm}$ from the opening of the tube on subsequent tool selections to retrieve candies $16 \mathrm{~cm}$ from the opening, and vice versa. The third result was related to the major purpose of Experiment 1a, and showed that although the participants were most likely to experiment with different sticks early in training, this experimentation did not cause people to select tools whose lengths varied significantly or systematically across trials. Within the parameters of the present experiment, it seems that experience retrieving an object does not cause adult humans to select a tool whose length more closely matches the distance over which an object has to be retrieved. Instead, whatever adjustments occurred were minor and did not vary systematically across participants.

Exactly why the participants in this experiment and others (e.g., Silva \& Silva, 2010) have not matched object distance to stick length remains unclear. Our guess is that choosing a stick whose length matches the object's distance simply does not provide any room for error in judging the object's distance or using the tool to retrieve the object, an explanation also advanced by other researchers (e.g., Hunt et al., 2006) and consistent with some participants' explanations for their choices. When participants were asked to explain how their experience retrieving the candy affected the stick they selected, most of them reported something about the stick's length and usability. For example, at the 8 -cm distance one person reported, "I tried to pick the one [stick] that was most efficient - not too short but not too long. The short ones probably would have gotten the job done, but I wanted one with a little more length in case something went wrong and I'd have a bit of recovery room." At the 16-cm distance, this person said, "I knew right away that the shorter sticks weren't going to do the job, and I thought maybe the middle one would do what I needed it to do. I felt the longest stick was probably too long and would most likely push the object away, making it difficult to retrieve." In a previous study, participants given one trial to retrieve a candy from the open end of a tube gave similar explanations (see Silva \& Silva, 2010). Whether the participants' explanations reflect their evaluation of the problem before they selected a stick or are self-justifying narratives made after the fact (object retrieval) is unknown. In some tooluse problems, adult humans' explanations for their tool selections and how they used them were incongruent with what they actually did (for an example and a discussion of the implications of these incongruences for the study of physical causality, see Silva, Silva, Cover, Leslie, \& Rubalcaba, 2008).

But why, with repeated trials, did the participants not select tools whose lengths more closely matched the distance over which the desired object had to be retrieved? In some cases, the alternatives did not seem as good (in terms of ease of use, for example) as the previous selection. In other cases, a selection was "good enough," in that the object was successfully retrieved. Consider the following explanations offered by a participant: "I used longer sticks that I knew would reach the object and tried to use a smaller one in one instance and had trouble so I switched back to longer ones." Another participant said, "Since it [the candy] was in the same spot each time, I used the same stick because it successfully reached the item. I never chose a longer stick or a smaller stick since I knew that anything smaller might not reach and anything longer was excessive." These sorts of explanations suggest that rather than select a tool whose length more closely matched the distance over which an object had to be retrieved, the participants' selections across trials accorded with a "if successful, select the same or a similar tool on the next trial" strategy. This analysis fits with what has been observed in laboratory crows. For these animals, tool swapping usually takes the 
form of exchanging shorter tools for longer ones when the short tool is not long enough to reach the desired object (Wimpenny et al., 2009). In the present experiment, because the candy was successfully retrieved on the first trial, there was no need to select a different tool on subsequent trials.

Other possible reasons for the absence of matching or a propensity for choosing the longest stick may relate to differences in motivation between humans and crows, the threat (or lack thereof) of predation while trying to obtain the out-of-reach object, and the physical dimensions of the task. For example, while foraging in their natural environment or completing a tool-use task in the laboratory, crows are presumably working for a highly desirable food item. It is unlikely that the candies used with the human participants were as equally desired. In the present experiment, the participants most likely retrieved the candy simply because they were asked to do so. Moreover, it is likely that the foraging behavior of many animals, including crows, is modulated by uncertainty related to predator risk (see, e.g., Ferrari, Brown, Bortolotti, \& Chivers, 2010; Polo, López, \& Martín, 2011). No such threat would be present in the task presented to humans, though other concerns might be (e.g., embarrassment elicited by failing to retrieve the candy). In relation to the dimensions of the task, the lengths of the sticks that participants selected may have depended on the scale of the problem. Using sticks that are thicker, longer, and heavier than those used in the present study and using these to retrieve, for example, a baseball from a $30-\mathrm{cm}$ diameter pipe might produce different results.

Finally, the results of Experiment 1a showed that the orientation of the opening of the tube did not affect the length of the stick that the participants selected. Because the participants could walk around to any side of the table to retrieve the candy, the influence of someone's handedness on their stick selection may have been minimized. Had participants viewed the orientation of the opening as an obstacle (as right-handed people might if the opening faced left, and vice versa), they might have selected a longer stick when the opening's orientation was incongruent with their handedness. This appears not to have happened.

\section{Experiment 1b}

Overall, Experiment 1a showed that with repeated opportunities to select a tool and retrieve an object, adult humans do not come to select tools whose lengths more closely approximate the object's distance. Instead, participants judged the distance of an object, selected a stick that was long enough to reach the object, and then continued to select the same or a similar stick on subsequent trials. Although the participants' choices of sticks did not change systematically across trials, it is possible that they adjusted how they held a stick so that its working length changed across trials.

Experiment $1 \mathrm{~b}$ replicated Experiment 1a with one modification: After each trial, we measured the working length of the sticks selected by the participants, to determine whether they were adjusting the working length of the stick across trials. We did not measure working length in Experiment 1a because we were concerned that the act of measuring it might draw the participants' attention to this dependent measure and produce a subject-expectancy effect that influenced their selections or how they held the stick after the first trial.

\section{Method}

Participants A group of 20 undergraduate students attending a liberal arts college in southern California volunteered for the study and received partial course credit for their involvement.

Materials and procedure The same materials and procedure were used as in Experiment 1a, except that the experimenter measured the working length of each stick selected by a participant. The participants were instructed as in Experiment 1a to select a stick and to hold it in preparation to retrieve the candy. When a participant said that he or she was ready to retrieve the candy, the experimenter measured the distance between the working tip of the stick (i.e., the tip that would contact the candy) and the tip of the participant's finger closest to the working tip. This distance was the working length of the stick. The data analyses were similar to those used in Experiment 1a.

\section{Results and discussion}

Every participant retrieved the candy on every trial. Table 2 shows the distribution of the participants' tool selections. As in Experiment 1a, the participants' selections were not equally distributed across the 10 sticks, and thus not random. In contrast to Experiment 1a, the participants' selections were not as tightly clustered. When the candy's distance was $8 \mathrm{~cm}$ from the open end of the tube, the participants in Group 8-16 tended to select sticks that were 14-20 cm long and $24 \mathrm{~cm}$ long; the participants in Group 16-8 generally chose sticks that were $12-18 \mathrm{~cm}$ long. When the candy's distance was $16 \mathrm{~cm}$, the participants in Groups 8-16 and 16-8 most often selected sticks that were $18-26 \mathrm{~cm}$ long.

Figure 3 shows the average lengths of the sticks selected by the participants on each trial when the candy was 8 or $16 \mathrm{~cm}$ from the open end of the tube. The results were similar to those of Experiment 1a. That is, there was no overall difference between the two groups $[F(1,16)=2.16, p=.161]$, no effect of the orientation of the opening of the tube $[F(1,16)=0.65$, 
Table 2 Frequency distribution of sticks selected in Experiment 1b

\begin{tabular}{|c|c|c|c|c|c|c|c|c|c|c|c|}
\hline \multirow[t]{2}{*}{ Candy Distance } & \multirow[t]{2}{*}{ Trial } & \multicolumn{10}{|c|}{ Stick Length $(\mathrm{cm})$} \\
\hline & & 8 & 10 & 12 & 14 & 16 & 18 & 20 & 22 & 24 & 26 \\
\hline \multicolumn{12}{|l|}{ Group 8-16 } \\
\hline \multirow[t]{4}{*}{$8 \mathrm{~cm}$} & 1 & 0 & 0 & 2 & 1 & 3 & 2 & 1 & 0 & 1 & 0 \\
\hline & 2 & 0 & 1 & 0 & 2 & 2 & 1 & 1 & 1 & 2 & 0 \\
\hline & 3 & 0 & 0 & 1 & 2 & 2 & 3 & 1 & 0 & 1 & 0 \\
\hline & 4 & 0 & 0 & 1 & 2 & 1 & 2 & 2 & 0 & 2 & 0 \\
\hline \multirow[t]{4}{*}{$16 \mathrm{~cm}$} & 5 & 0 & 0 & 0 & 0 & 0 & 2 & 2 & 1 & 3 & 2 \\
\hline & 6 & 0 & 0 & 0 & 0 & 2 & 1 & 2 & 0 & 2 & 3 \\
\hline & 7 & 0 & 0 & 0 & 0 & 0 & 1 & 2 & 0 & 4 & 3 \\
\hline & 8 & 0 & 0 & 0 & 0 & 0 & 1 & 2 & 2 & 3 & 2 \\
\hline \multicolumn{12}{|l|}{ Group 16-8 } \\
\hline \multirow[t]{4}{*}{$16 \mathrm{~cm}$} & 1 & 0 & 0 & 0 & 0 & 0 & 3 & 3 & 1 & 3 & 0 \\
\hline & 2 & 0 & 0 & 0 & 0 & 0 & 3 & 1 & 2 & 2 & 2 \\
\hline & 3 & 0 & 0 & 0 & 0 & 0 & 2 & 2 & 1 & 2 & 3 \\
\hline & 4 & 0 & 0 & 0 & 0 & 0 & 5 & 2 & 2 & 1 & 0 \\
\hline \multirow[t]{4}{*}{$8 \mathrm{~cm}$} & 5 & 0 & 0 & 3 & 2 & 1 & 3 & 0 & 0 & 0 & 1 \\
\hline & 6 & 0 & 0 & 1 & 1 & 1 & 6 & 0 & 1 & 0 & 0 \\
\hline & 7 & 0 & 0 & 1 & 1 & 2 & 5 & 0 & 0 & 0 & 1 \\
\hline & 8 & 0 & 0 & 1 & 3 & 3 & 3 & 0 & 0 & 0 & 0 \\
\hline
\end{tabular}

$p=.431]$, no difference across trials $[F(3,48)=1.23, p=.311]$, and no interactions among any combinations of the factors $\left[F_{\mathrm{s}}(1,16)<0.51, p \mathrm{~s}>.487\right.$, and $\left.F_{\mathrm{s}}(3,48)<2.02, p \mathrm{~s}>.124\right]$. Thus, for both groups at both of the candy's distances, tool selections did not change across trials. The only difference shown in Fig. 3 is that the average lengths of the sticks chosen by the participants were shorter when the candy's distance was $8 \mathrm{~cm}(M=17.0 \mathrm{~cm}, S D=3.59)$ than when it was $16 \mathrm{~cm}$ $(M=21.8 \mathrm{~cm}, S D=3.06), F(1,16)=47.64, p<.001$.

Figure 4 shows the average working lengths of the sticks used by the participants on each trial for both of the candy's distances. Although the working lengths were necessarily

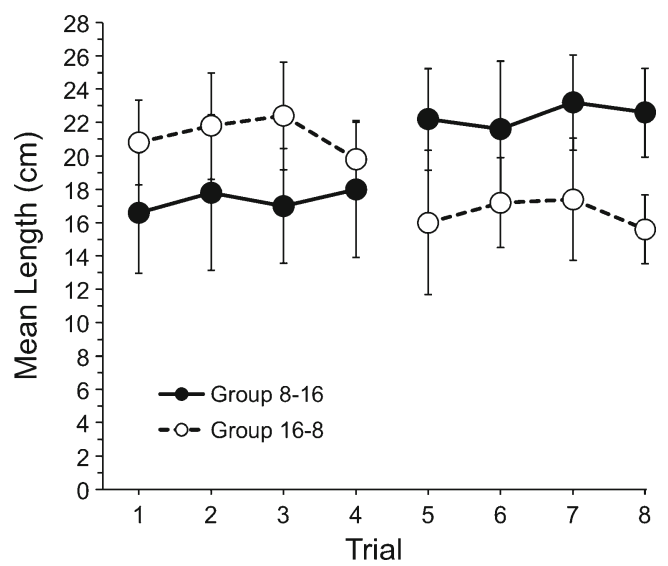

Fig. 3 Mean lengths of the sticks selected in Experiment $1 \mathrm{~b}$ for each group across all trials. The details are the same as for Fig. 2 shorter than the sticks' actual lengths, the overall pattern of results was the same as for the actual lengths. That is, there was no difference between the two groups $[F(1,16)=0.02$, $p=.882]$, no effect of the orientation of the opening of the tube $[F(1,16)=0.344, p=.565]$, no difference across trials $[F(3,48)=1.00, p=.403]$, and, with two exceptions, no interactions among most combinations of the factors $\left[F_{\mathrm{s}}(1,16)<0.38, p \mathrm{~s}>.548\right.$, and $F \mathrm{~s}(3,48)<3.18$, $p \mathrm{~s}>.032]$. The exceptions were a significant interaction between the orientation of the opening of the tube and trial $[F(3,48)=3.18, p=.032]$ and an interaction among all four factors $[F(3,48)=2.82, p=.049]$. Post hoc analyses of the

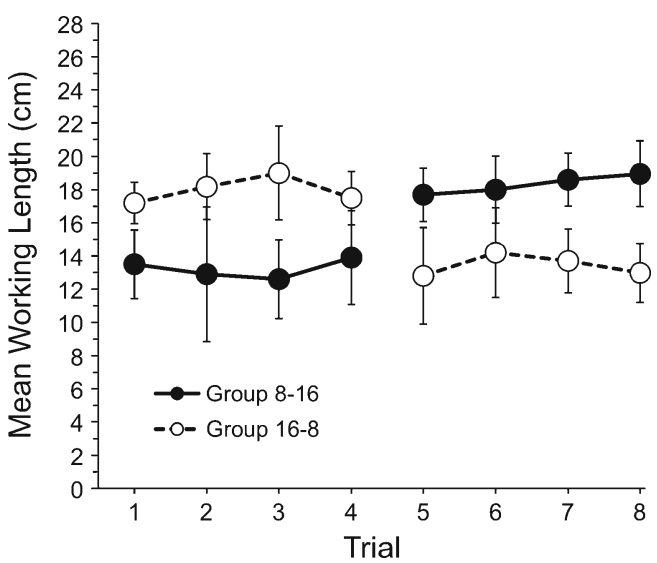

Fig. 4 Mean working lengths of the sticks selected in Experiment $1 \mathrm{~b}$ for each group across all trials. The details are the same as for Fig. 2 
significant interactions did not reveal any meaningful pattern to these interactions. For example, there were no significant pairwise interactions between the orientation of the opening of the tube and trial $(p s>.182)$. In sum, for both groups and both of the candy's distances, the working lengths of the sticks used to retrieve the candy did not change across trials. The only difference shown in Fig. 4 is that the average working length of the stick used by the participants was shorter when the distance of the candy was $8 \mathrm{~cm}(M=13.3 \mathrm{~cm}$, $S D=2.61)$ than when it was $16 \mathrm{~cm}(M=18.1 \mathrm{~cm}, S D=1.93)$, $F(1,16)=115.83, p<.001$.

As in Experiment 1a, we looked again at whether the participants selected a tool that was different from the one they had selected on the previous trial. Collapsing across the candy's two distances, the results showed that on Trials 2, 3, and $4,80 \%, 65 \%$, and $55 \%$ of the participants, respectively, selected a different stick from the one they had chosen on the previous trial. The candy's distance from the opening of the tube was changed before the fifth trial. On Trials 6, 7, and 8,55\%, 45\%, and $25 \%$ of the participants selected a different stick from the one they had chosen on the previous trial. As in Experiment 1a, the participants were most likely to select a different stick between Trials 1 and 2, and then to swap sticks less often across trials. Cochran's $Q$ test confirmed that there was a statistically significant difference in the percentage of participants who selected a different stick across the six pairs of trials noted above $[Q(5)=13.32, p=.021]$.

We also conducted a similar analysis of the working lengths of the sticks. Because working lengths were measured in resolutions of $1 \mathrm{~mm}$, even a change from, for example, a working length from 15.9 to $16.1 \mathrm{~cm}$ could be considered a change in working length. To avoid counting every variation in how the participants held a stick as a change in its working length, we defined "change in working length" as a difference of $2 \mathrm{~cm}$ or more in the working lengths of the sticks selected on two successive trials. This length (i.e., $2 \mathrm{~cm}$ ) was the same as the smallest difference in length between any two pairs of sticks.

Collapsing across the candy's two distances, the results showed that on Trials 2, 3, and $4,55 \%, 40 \%$, and $35 \%$ of the participants, respectively, changed the working length of the stick from that of the previous trial. The candy's distance from the opening of the tube was changed before the fifth trial. On Trials 6,7 , and $8,50 \%, 45 \%$, and $15 \%$ of the participants changed the working lengths of their sticks from the length on the previous trial. The percentages of participants who adjusted the working lengths of the sticks by a sufficient amount (as per the operational definition) did not change significantly across these six pairs of trials $[Q(5)=8.22$, $p=.145]$. (As an aside, the same pattern was evident when we defined "change in working length" as a difference of $1 \mathrm{~cm}$ or more in the working lengths of the sticks selected on two successive trials.) Although the percentages of participants who adjusted the working lengths of the sticks across the six trials were not statistically significant, the difference between the percentages of people who adjusted the working length between Trials 1 and $2(55 \%)$ and Trials 7 and $8(15 \%)$ was statistically significant $[Q(1)=5.33, p=.021]$.

The results of Experiment 1b replicated those of Experiment 1a. The length of the stick selected by the participants was influenced by the candy's distance from the opening of the tube, was unaffected by having retrieved candies at another distance, and did not change as a function of experience retrieving the candy. Moreover, although the participants were most likely to experiment with different sticks early in training, this experimentation did not produce tool selections whose lengths changed significantly and systematically across trials. Most importantly for the purpose of Experiment 1b, the same results also applied to the working lengths of the sticks that the participants used. This means that the participants' tool selections were influenced by how the tool would be used or held; otherwise, the working lengths of the tools selected would have occasionally been too short to reach the candy. By selecting tools whose working lengths were always sufficient to reach the candy, the participants showed that they must have considered how a stick would be held or used.

\section{Experiment 2}

Experiments $1 \mathrm{a}$ and $1 \mathrm{~b}$ showed that, with repeated trials, the participants did not eventually select a tool whose actual length or working length more closely approximated the distance over which an object had to be retrieved. In short, experience with the tools and the task did not cause the participants' tool selections to become more like those observed in some New Caledonian crows (e.g., Chappell \& Kacelnik, 2002). In Experiment 2, we focused on the other result observed in some crows-namely, that they select the longest stick in a set to retrieve an object in a tube (e.g., Chappell \& Kacelnik, 2002).

Adult humans do not generally select the longest stick in a set to retrieve an out-of-reach object (Silva \& Silva, 2010). Instead, people select a stick that is long enough to reach the object but not so long as to be unnecessarily difficult to use. This strategy affords people some room for error, such as misjudging the object's distance or inadvertently pushing the object farther into the tube, while avoiding the costs associated with using a tool that is unnecessarily long. In Experiment 2, we tested whether this margin for error would increase if the problem were more ambiguous.

If the stick that people select is influenced by some degree of uncertainty (related to judging lengths and distances or how to use the tool), then making a problem more 
ambiguous should increase this uncertainty. People who are uncertain about the best stick to use to solve the problem should select a longer, safer stick than do people who are more certain about what to do. Perhaps under very ambiguous situations, people may behave like Chappell and Kacelnik's (2002) crows and select the longest tool in a set. In Experiment 2, we compared the tool selections of a group of people who had unlimited time to study the problem with those of a group who had only $1 \mathrm{~s}$ to do the same.

\section{Method}

Participants and materials A group of 40 undergraduate students attending a liberal arts college in southern California volunteered for the study and received partial course credit for their involvement. The same materials were used as in Experiments $1 \mathrm{a}$ and $1 \mathrm{~b}$, with the following addition: The privacy glass was operational, in the sense that it was sometimes opaque and sometimes transparent (see the Procedure section below). When unpowered, the opaque privacy glass prevented participants from seeing the tube and the candy.

Procedure The same general procedure from Experiments $1 \mathrm{a}$ and $1 \mathrm{~b}$ was used here, with the following exceptions. The experimenter began by asking a participant to enter the room and to stand in front of the tube and sticks while the experimenter read instructions similar to those used in the previous experiments. In addition, the participants were told that they would see an opaque glass "screen" above the tube when they reentered the room, that this glass would prevent them from seeing the tube and the candy, and that at the appropriate time the glass would clear so that they could see the tube and the candy. While the procedure was explained to the participants, the privacy glass was not yet positioned above the tube, nor was there any candy in the tube. The experimenter then asked the participant to leave the room while the candy was positioned in the tube and the glass was placed over the tube and candy.

There were 10 participants in each of four groups: a group for whom the candy was placed $8 \mathrm{~cm}$ from the open end of the tube and who were given a limited amount of time to study the tube and candy (Group Limited-8); a similar group for whom the candy's distance was $16 \mathrm{~cm}$ (Group Limited-16); a group that had an unlimited amount of time to study the candy placed $8 \mathrm{~cm}$ from the opening of the tube (Group Unlimited-8); and a similar group for whom the candy's distance was $16 \mathrm{~cm}$ (Group Unlimited-16).

For the two limited groups, the participants were instructed to look down at the opaque glass when they reentered the room. When a participant said that she or he was ready, the experimenter cleared the glass for $1 \mathrm{~s}$, after which it switched back to its opaque state. As in Experiment 1b, the participants were then asked to select a stick and to hold it as if they were ready to make their attempt to retrieve the candy. The experimenter then measured the stick's working length before clearing the privacy glass again so the participant could retrieve the candy. For the two unlimited groups, the procedure was the same, except that the privacy glass never returned to its opaque state (i.e., once cleared, it remained clear).

All other aspects of the procedure were the same as in Experiments 1a and 1b, except that there was only a single trial. We were interested in four pairwise between-groups comparisons for each dependent measure (stick length and working length) - Groups Limited-8 versus Unlimited-8, Limited-16 versus Unlimited-16, Limited-8 versus Limited16 , and Unlimited-8 versus Unlimited-16. Unless otherwise stated, the results were analyzed with an ANOVA consisting of three between-groups factors (amount of time to evaluate the problem, candy's distance, and orientation of the opening of the tube). Tukey's HSD test was used for the post hoc analyses. For all analyses, $\alpha=.05$ was used to determine statistical significance.

\section{Results and discussion}

Every participant retrieved the candy. Table 3 shows the distribution of the participants' tool selections. Group Limited-8's selections were distributed mostly across the 18- to 22-cm sticks, and Group Unlimited-8's selections were distributed across the $12-$ to 20 -cm sticks. Group Limited-16's selections consisted almost exclusively of the 26-cm stick, and Group Unlimited-16's selections were distributed mostly across the 18 - to 22 -cm sticks.

Table 3 Frequency distribution of sticks selected in Experiment 2

\begin{tabular}{llllllllll}
\hline Group & \multicolumn{2}{l}{ Stick Length $(\mathrm{cm})$} & & & & \\
\cline { 2 - 9 } & 8 & 10 & 12 & 14 & 16 & 18 & 20 & 22 & 24 \\
\hline Limited-8 & 0 & 0 & 0 & 0 & 1 & 4 & 2 & 2 & 0 \\
Unlimited-8 & 0 & 0 & 2 & 2 & 1 & 2 & 3 & 0 & 1 \\
Limited-16 & 0 & 0 & 0 & 0 & 0 & 0 & 0 & 0 & 0 \\
Unlimited-16 & 0 & 0 & 0 & 0 & 0 & 2 & 4 & 3 & 0 \\
\hline
\end{tabular}




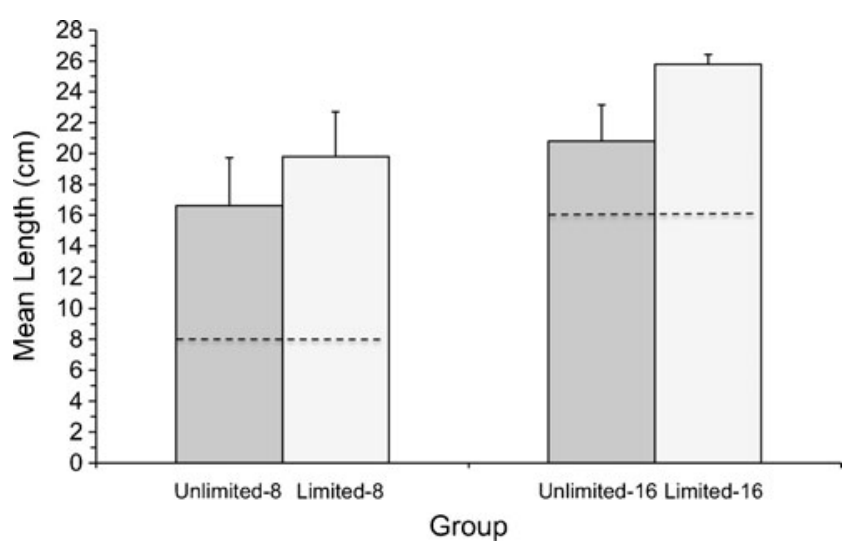

Fig. 5 Mean lengths of the sticks selected by the participants in Experiment 2. The error bars show standard deviations. The dashed lines across the bars show the distance of the candy from the open end of the tube

Figure 5 shows the average lengths of the sticks selected by the participants. There was no effect of the orientation of the tube $[F(1,32)=0.058, p=.811]$, but there were main effects of the amount of time to study the problem $[F(1,32)=25.56$, $p<.001]$ and the candy's distance $[F(1,32)=39.19, p<.001]$. There were no interactions among any combinations of the factors $[F \mathrm{~s}(1,32)<0.93, p \mathrm{~s}>.343]$. When the candy was $8 \mathrm{~cm}$ from the opening of the tube, the average stick selected by Group Limited-8 $(M=19.8 \mathrm{~cm}, S D=2.90)$ was significantly longer than the average stick selected by Group Unlimited-8 $(M=16.4 \mathrm{~cm}, S D=3.23), p=.032$. Similarly, when the candy was $16 \mathrm{~cm}$ from the opening of the tube, the average length of the stick selected by Group Limited-16 $(M=25.8 \mathrm{~cm}, S D=$ $0.63)$ was significantly longer than the average stick selected by Group Unlimited-16 $(M=20.8 \mathrm{~cm}, S D=2.35), p=.001$.

Figure 6 shows the average working lengths of the sticks used by the participants. There was no effect of the orientation of the tube $[F(1,32)=0.46, p=.503]$, but there were main effects of the amount of time to study the problem $[F(1,32)=$ $4.68, p=.040]$ and the candy's distance $[F(1,32)=72.42$,

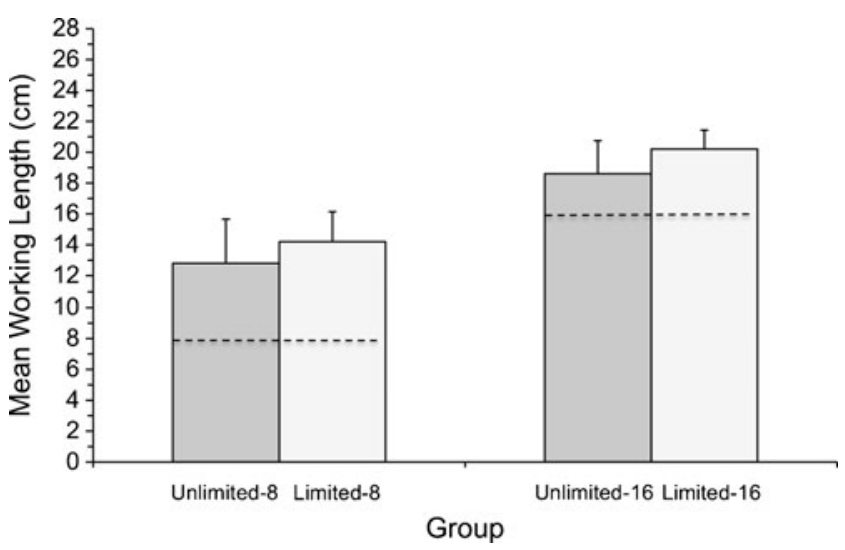

Fig. 6 Mean working lengths of the sticks selected by the participants in Experiment 2. The details are the same as for Fig. 5 $p<.001]$. There were no interactions among any combinations of the factors $[F \mathrm{~s}(1,32)<0.58, p \mathrm{~s}>.450]$. When the candy's distance was $8 \mathrm{~cm}$, the average working length of the sticks used by Group Limited-8 $(M=14.2 \mathrm{~cm}, S D=2.16)$ was similar to the average working length of the sticks used by Group Unlimited-8 $(M=12.8 \mathrm{~cm}, S D=2.86), p=.492$. The same was true when the candy's distance was $16 \mathrm{~cm}$ : The average working length of the sticks used by Group Limited$16(20.2 \mathrm{~cm}, S D=1.23)$ was similar to the average working length of the sticks used by Group Unlimited-16 $(18.6 \mathrm{~cm}$, $S D=1.94), p=.376$.

Comparing the sticks selected when the candy's distance from the opening of the tube was 8 versus $16 \mathrm{~cm}$ showed that the participants selected a shorter stick at the shorter distance. This effect was observed regardless of whether the participants had a limited (Groups Limited-8 vs. Limited-16, $p<.001$ ) or an unlimited (Groups Unlimited-8 vs. Unlimited-16, $p=.002$ ) amount of time to study the task. The same was true of the average working lengths of the sticks used by the participants (Unlimited-8 $<$ Unlimited-16, $p<.001$; Limited-8 $<$ Limited$16, p<.001)$.

The results showed that limiting the amount of time that participants could study the task led them to select a longer stick than those selected by the participants who had an unlimited amount of time to study the task. Presumably, limiting how long the participants examined the task increased their uncertainty about it, which may have influenced them to err on the side of caution and to select a longer stick. This sort of selection pressure may be similar to what Mechner (1958) observed when rats had to switch from pressing one lever to pressing another after completing a response requirement (e.g., pressing the first lever 16 times). If a rat switched to the second lever too early, it did not receive reinforcement and it had to start from the beginning and make another, for example, 16 responses on the first lever before reinforcement on the second lever became available. Under this selection pressure, the rats produced run lengths that were generally longer than required. This strategy, which could be described as erring on the side of caution, prevented the unwanted penalty for switching too early.

Some of the participants' explanations for their selections are consistent with this analysis. For example, one person in Group Limited-16 said, "The object was in the middle, so I needed a long stick to make sure that I got it." Another participant in this same group said, "The longest makes sense to have more tool to use. I wasn't sure how far it [the candy] was, and if I hit it farther, I could still get it."

The foregoing analysis is also consistent with the results of the sticks' working lengths. Recall that the average working length of the stick selected depended on the candy's distance, such that longer sticks were selected when the candy was farther from the opening of the tube. However, 
within a particular distance of the candy from the opening, the average working length of the sticks selected was unaffected by the amount of time participants had to study the problem. Thus, Groups Limited- 8 and Unlimited- 8 used sticks of similar working lengths, as did Groups Limited16 and Unlimited-16. This suggests that limiting the amount of time that the participants could evaluate the problem influenced them to select a longer stick (i.e., Limited- $8>$ Unlimited-8 and Limited-16 > Unlimited-16). But once the participants had made their selection and the privacy glass was cleared so that they could retrieve the candy, the participants in the limited groups may have realized that the stick they selected was unnecessarily long, at which time they adjusted how they held the stick so that its working length was similar to those used by the participants in the corresponding unlimited group (i.e., Limited-8 $=$ Unlimited8 and Limited-16 = Unlimited-16).

In sum, Experiment 2 showed that people select a longer tool, although not necessarily the longest tool in a set, when a tool-use situation is more ambiguous. Although the participants in the limited groups selected longer sticks than did the participants in the unlimited groups, whether the participants in the limited groups selected the longest stick depended on the candy's distance. In Group Limited-8, only one of the 10 participants selected the longest stick. In Group Limited-16, however, nine of the 10 participants selected the longest stick. Thus, it seems that ambiguity about a task influenced participants to select the longest tool only when the candy's distance from the open end of the tube was relatively close to the length of the longest stick. This result for Group Limited-16 bears some resemblance to that of a New Caledonian crow that tended to select longer sticks when the stick-and-tube task became more demanding by placing the tube and tools farther apart and separating them with a barrier (Chappell \& Kacelnik, 2002).

The difference in the mean lengths of the sticks selected by the participants in Groups Limited- 8 versus Limited- 16 tells us that, even under conditions of more uncertainty, these people did not use an isolated "choose-the-longeststick" strategy. If they had, both groups would have chosen the longest stick to retrieve the candy. That this did not happen suggests that most of the participants in Group Limited-16 chose the longest $(26-\mathrm{cm})$ stick because this stick was within the range of suitable lengths, perhaps even the ideal length, given the physical dimensions of the tools and the task and the constraints placed on evaluating it (see the discussion in Silva \& Silva, 2010). Had the longest stick been, say, $60 \mathrm{~cm}$, it seems unlikely that the participants would have chosen it. We wonder whether the same might be true of the crows that have been observed selecting the longest stick in the set. Is the fact that some crows choose the longest stick in a set an artifact of the range of stick lengths?

\section{General discussion}

The results of Experiments $1 \mathrm{a}$ and $1 \mathrm{~b}$ showed that decreasing participants' uncertainty about a tool-use task by allowing them to select tools and retrieve an object across several trials did not affect the length of the tool that they selected. Although the participants' tool selections in Experiments 1a and $1 \mathrm{~b}$ were related to the distance of an out-of-reach object, participants did not systematically vary their selections or how they held the tools across trials. The participants generally selected a stick that was long enough to reach the object, but not necessarily the longest stick in the set. Moreover, experience retrieving an object at one distance did not influence tool selection when the object's distance from the opening of the tube was doubled (for Group 8-16) or halved (for Group 16-8). The participants' selections across trials accorded with a "win-stay" strategy, though more participants did experiment with (swap) tools in earlier than in later trials.

Although some studies of New Caledonian crows have suggested that these animals tend to select tools whose lengths match the distance of the task requirement (Chappell $\&$ Kacelnik, 2002), other studies have found little evidence of this tendency (Hunt et al., 2006; Wimpenny et al., 2009). The reasons for the different results may be due to variations in the apparatus (e.g., laboratory stick-and-tube problem vs. natural foraging sites), procedures (e.g., presenting crows with sticks that are always organized from shortest to longest vs. allowing crows to find their own leaf-tools), and the birds' experiences (e.g., more vs. less experience with stick-tools) (see also Chappell \& Kacelnik, 2002; Taylor, Elliffe, Hunt, \& Gray, 2010; Taylor, Hunt, Medina, \& Gray, 2009). In the case of adult humans, we can rule out lack of experience with the tools and task as a reason for their not matching tool length to object distance.

Despite the lack of evidence for matching, it may be unreasonable to expect anyone to precisely match the actual or working length of a stick to an object's distance. In the same sense that a fair coin tossed 100 times is unlikely to produce exactly 50 "heads," it might also be unlikely that people or other animals would exactly match the length of a tool or its working length to the distance of a desired object. In Experiment 1b, the working lengths of the sticks selected did not deviate much - dependent, of course, on someone's definition of "much"-from the candy's distance. The mean working lengths of the sticks selected were 13.3 and $18.1 \mathrm{~cm}$ when the candy was 8 and $16 \mathrm{~cm}$, respectively, from the open end of the tube. Whether these deviations from matching are close enough to conclude that humans have a tendency to match the working lengths of a tool to the distance of a desired object is subjective. One thing is certain from Experiments $1 \mathrm{a}$ and $1 \mathrm{~b}$ : Within the parameters of these experiments, increased experience 
with the tools and task did not cause the participants to select progressively shorter sticks. Stated another way, a decrease in uncertainty (resulting from having multiple opportunities to solve a problem) did not influence participants to select tools whose lengths approached the distance of an outof-reach object.

In contrast, Experiment 2 showed that limiting how much time the participants had to study a tool-use task led them to select longer sticks than did people allowed unlimited time to study the task. Stated another way, an increase in uncertainty about a task (resulting from limiting how long someone could study the problem) influenced participants to select longer tools. It is tempting to wonder whether crows' tool selections might be influenced by this same variable.

Assuming that uncertainty about a problem is inversely related to a subject's experience solving similar problems, one might guess that inexperienced crows are more likely than experienced crows to select longer tools. In reality, though, less experienced crows are more likely than experienced crows to select tools that are too short to retrieve out-ofreach food (Wimpenny et al., 2009). And, regardless of their experience, crows that select a tool that is too short to reach the food will generally select a longer tool on subsequent trials (Wimpenny et al., 2009). This result suggests that some crows understand that longer, not shorter, tools are necessary after a failed attempt to reach a desired object. How this understanding comes about is unclear. It could be the result of trial-anderror and learned associations between shorter sticks paired with failure and longer sticks paired with success. In some instances, though, rooks (Corvus frugilegus) seem to solve physical causality problems "using advanced physical cognition rather than trial-and-error learning" (Bird \& Emery, 2010, p. 150), and New Caledonian crows solve tool-use tasks without explicit training to do so (Taylor et al., 2010; Weir, Chappell, \& Kacelnik, 2002). What role learning plays in the formation of advanced physical cognition or the ability to solve a problem without explicit training remains to be investigated and debated (Machado \& Silva, 2003; Shettleworth, 2010; Tomasello \& Call, 1997).

What we can say is that the absence of matching stick length to object distance in the present study and a previous study (Silva \& Silva, 2010) is not the result of people's inexperience with the tools or task. We can also say that the participants' tool selections were influenced by the following variables: distance of the desired object, a sensitivity to how the tool would be held or used, and uncertainty about the task. What we cannot say at this time is the extent to which these results depend on the ergonomics of the task and the tools. The length of the sticks that participants select may depend on the scale of the problem. Perhaps if the sticks and the candy's distance were close to adult humans' body lengths and the weight of the sticks were closer to the limits of what the participants could hold comfortably, the results might have been more similar to those reported with New Caledonian crows. Specifically, a greater cost of using an unnecessarily long stick might influence people to select a stick whose length more closely matches the object's distance. Other procedural variables may also have contributed to the results. For example, in the procedure we used, the out-ofreach object could be pushed deeper into the tube. A revised procedure consisting of a barrier placed behind the candy that precluded this possibility might produce different stick selections from those reported above. Studies with great apes have documented the importance of whether an animal has to push or pull a tool to reach a desired object (Girndt, Meier, \& Call, 2008). Finally, as we discussed in Experiment 1a, motivational variables related to the out-of-reach object and risks from threats while attempting to retrieve it may also account for the differences between humans and crows.

These unresolved issues notwithstanding, we can form tentative conclusions based on the results of the present study. First, it does not appear that inexperience is the cause for the lack of matching stick length to object distance observed in adult humans. The participants in Experiments $1 \mathrm{a}$ and $1 \mathrm{~b}$ did not systematically select, across multiple trials, sticks whose lengths became more similar to the candy's distance. Second, it is clear that an object's distance influenced the participants' tool selections. If it had been otherwise, the participants would have selected sticks of similar lengths, regardless of the candy's distance. Third, it is also clear that the participants' tool selections were influenced by how the tool would be used or held. If the participants had not considered how they would hold a stick or that some portion of the stick must necessarily be unusable to extend their reach (because some portion of the tool is in the hand instead of in the tube), they should have occasionally selected tools whose working lengths were too short to reach the candy, something that never occurred. Fourth, it seems that increased uncertainty about the task, which was produced by limiting how much time the participants could study it, inclined participants to select longer sticks than did participants who were allowed unlimited time to do the same. If uncertainty had not been a factor, the two groups of participants would have selected sticks of similar lengths. Finally, it is unlikely that adult humans use a "choosethe-longest-stick" strategy under conditions of uncertainty (at least within the parameters of the experiments reported here). If they had, the average lengths of the sticks selected by Groups Limited- 8 and Limited-16 would have been the same. Overall, this study adds to an increasing list of studies and researchers who have argued that the structural and procedural features of tool-use problems are important, and that researchers need to be cautious when forming conclusions about a species' cognitive abilities (Girndt et al., 2008; Seed, Call, Emery, \& Clayton, 2009; Shettleworth, 2010; Silva et al., 2005). 
Author Note We thank the action editor and two anonymous reviewers for their helpful critiques, and Armando Machado for his invaluable comments and discussions about a previous draft of this article and about the statistical analyses.

\section{References}

Bird, C. D., \& Emery, N. J. (2010). Rooks perceive support relations similar to six-month-old babies. Proceedings of the Royal Society B, 277, 147-151. doi:10.1098/rspb.2009.1456

Bluff, L. A., Troscianko, J., Weir, A. A. S., Kacelnik, A., \& Rutz, C. (2010). Tool use by wild New Caledonian crows Corvus moneduloides at natural foraging sites. Proceedings of the Royal Society B, 277, 1377-1385. doi:10.1098/rspb.2009.1953

Chappell, J., \& Kacelnik, A. (2002). Tool selectivity in a non-primate, the New Caledonian crow (Corvus moneduloides). Animal Cognition, 5, 71-78. doi:10.1007/s10071-002-0130-2

Emery, N. J., \& Clayton, N. S. (2009). Tool use and physical cognition in birds and mammals. Current Opinion in Neurobiology, 19, 27 33. doi:10.1016/j.conb.2009.02.003

Ferrari, M. C. O., Brown, G. E., Bortolotti, G. R., \& Chivers, D. P. (2010). Linking predator risk and uncertainty to adaptive forgetting: A theoretical framework and empirical test using tadpoles. Proceedings of the Royal Society B, 277, 2205-2210. doi:10.1098/rspb.2009.2117

Girndt, A., Meier, T., \& Call, J. (2008). Task constraints mask great apes' ability to solve the trap-table task. Journal of Experimental Psychology: Animal Behavior Processes, 34, 54-62. doi:10.1037/ 0097-7403.34.1.54

Hunt, G. R., Rutledge, R. B., \& Gray, R. D. (2006). The right tool for the job: What strategies do wild New Caledonian crows use? Animal Cognition, 9, 307-316. doi:10.1007/s10071-006-0047-2

Machado, A., \& Silva, F. J. (2003). You can lead an ape to a tool, but. .. : A review of Povinelli's Folk physics for apes: A chimpanzee's theory of how the world works. Journal of the Experimental Analysis of Behavior, 79, 267-286. doi:10.1901/jeab.2003.79-267

Mechner, F. (1958). Probability relations within response sequences under ratio reinforcement. Journal of the Experimental Analysis of Behavior, 1, 109-121. doi:10.1901/jeab.1958.1-109

Polo, V., López, P., \& Martín, J. (2011). Uncertainty about future predation risk modulates monitoring behavior from refuges in lizards. Behavioral Ecology, 22, 2018-223. doi:10.1093/beheco/arq065
Povinelli, D. J. (2000). Folk physics for apes: The chimpanzee's theory of how the world works. New York, NY: Oxford University Press.

Seed, A. M., Call, J., Emery, N. J., \& Clayton, N. S. (2009). Chimpanzees solve the trap problem when the confound of tool-use is removed. Journal of Experimental Psychology: Animal Behavior Processes, 35, 23-34. doi:10.1037/a0012925

Shettleworth, S. J. (2010). Cognition, evolution, and behavior (2nd ed.). New York, NY: Oxford University Press.

Silva, F. J., \& Silva, K. M. (2006). Humans' folk physics is not enough to explain variations in their tool-using behavior. Psychonomic Bulletin \& Review, 13, 689-693. doi:10.3758/BF03193982

Silva, F. J., \& Silva, K. M. (2010). How do humans compare with New Caledonian crows in tool selectivity? Learning \& Behavior, 38, 87-95. doi:10.3758/LB.38.1.87

Silva, F. J., Page, D. M., \& Silva, K. M. (2005). Methodologicalconceptual problems in the study of chimpanzees' folk physics: How studies with adult humans can help. Learning \& Behavior, 33, 47-58. doi:10.3758/BF03196049

Silva, F. J., Silva, K. M., Cover, K. R., Leslie, A. L., \& Rubalcaba, M. A. (2008). Humans' folk physics is sensitive to physical connection and contact between a tool and reward. Behavioural Processes, 77, 327 333. doi:10.1016/j.beproc.2007.08.001

Taylor, A. H., Hunt, G. R., Medina, F. S., \& Gray, R. D. (2009). Do New Caledonian crows solve physical problems through causal reasoning? Proceeding of the Royal Society B, 276, 247-254. doi:10.1098/rspb.2088.1107

Taylor, A. H., Elliffe, D., Hunt, G. R., \& Gray, R. D. (2010). Complex cognition and behavioural innovation in New Caledonian crows. Proceedings of the Royal Society B, 277, 2637-2643. doi:10.1098/ rspb.2010.0285

Tebbich, S., \& Bshary, R. (2004). Cognitive abilities related to tool use in the Woodpecker Finch, Cactospiza pallida. Animal Behaviour, 67, 689-697. doi:10.1016/j.anbehav.2003.08.003

Tomasello, M., \& Call, J. (1997). Primate cognition. New York, NY: Oxford University Press.

Weir, A. A. S., Chappell, J., \& Kacelnik, A. (2002). Shaping of hooks in New Caledonian crows. Science, 297, 981. doi:10.1126/ science. 1073433

Wimpenny, J. H., Weir, A. A. S., Clayton, L., Rutz, C., \& Kacelnik, A. (2009). Cognitive processes associated with sequential tool use in New Caledonian crows. PLoS ONE, 4, e6471. doi:10.1371/journal. pone. 0006471 